\title{
Type $D$ personality as a predictor of self-efficacy and social support in patients with type 2 diabetes mellitus
}

This article was published in the following Dove Press journal:

Neuropsychiatric Disease and Treatment

20 March 2017

Number of times this article has been viewed

\section{Yechang Shao ${ }^{1,2}$ \\ Honglei Yin ${ }^{3}$ \\ Chengsong Wan ${ }^{4}$}

'School of Public Health, Southern Medical University, ${ }^{2}$ Department of Internal Medicine, Guangdong General Hospital, Guangdong Academy of Medical Sciences, ${ }^{3}$ Department of Psychiatry, Nanfang Hospital, ${ }^{4}$ Department of Microbiology, Guangdong Provincial Key Laboratory of Tropical Disease Research, School of Public Health, Southern Medical University, Guangzhou, People's Republic of China
Correspondence: Chengsong Wan Department of Microbiology, Guangdong Provincial Key Laboratory of Tropical Disease Research, School of Public Health, Southern Medical University, No 1023 Shatai Road, Guangzhou, 510515 , People's Republic of China Tel +862061648304 Email gzcswan@I63.com
Objective: The aim of this study was to estimate the prevalence of Type D personality and assess the relationship between this personality type and self-efficacy/social support in Chinese patients with type 2 diabetes mellitus (T2DM).

Patients and methods: From January 1, 2014, to July 31, 2014, 532 consecutive patients with T2DM were recruited from two hospitals in Guangzhou, China. The participants completed questionnaires containing questions about sociodemographic characteristics, Type D personality, self-efficacy, and social support scales, and their medical records were reviewed for additional data.

Results: Of the 532 patients, $18.2 \%$ had Type D personality. Patients with this personality type reported significantly lower levels of self-efficacy $(P<0.001)$, total social support $(P<0.001)$, subjective support $(P<0.001)$, and support utilization $(P=0.003)$, but similar level of objective support ( $P=0.314$ ), compared to those of patients without Type D personality. Negative affectivity and social inhibition, two intrinsic traits of Type D personality, negatively correlated with self-efficacy and social support scores. Type D personality was significantly associated with less self-efficacy and social support $(P<0.001)$, controlling for other sociodemographic factors. Glycosylated hemoglobin (HbA1c) levels were significantly higher in T2DM patients with Type D personality than in patients with non-Type D personality.

Conclusion: This study provides new evidence linking Type D personality with self-efficacy, social support, and poor glycemic control, highlighting the special need for care among T2DM patients with Type D personality.

Keywords: Type D personality, social support, self-efficacy, glycemic control, type 2 diabetes mellitus

\section{Introduction}

Diabetes is often accompanied with psychological distresses. There is a significantly higher prevalence of depression in patients with type 2 diabetes mellitus (T2DM) than in the general population, ${ }^{1,2}$ and their depressive symptoms have high rates of recurrence and chronicity. ${ }^{3}$ Furthermore, psychological disorders affect treatment choices, self-management, and short- and long-term outcomes in these patients. ${ }^{4}$ It is, therefore, vitally important to account for psychological factors in diabetes care.

Patient self-efficacy and social support are two concepts central to the psychological aspects of diabetes care. Patient self-efficacy is defined as the belief in one's capabilities to organize and execute the courses of action required to produce given attainments. ${ }^{5}$ Poor self-efficacy has been associated with increased depressive symptoms as well as poor glycemic control, ${ }^{6}$ and stronger self-efficacy has been associated with better 
self-management behaviors in diabetes, including control of dietary habits, exercise, blood sugar testing, and medication compliance. ${ }^{7,8}$ The evidence appears to be consistent that self-efficacy directly and indirectly influences diabetic therapeutic effectiveness and prognosis.

Social support is defined as the assistance received from others, which is a known buffer of psychological distress. ${ }^{9-12}$ It is measured in three dimensions: subjective support, objective support, and support utilization. Subjective support is the level of support a person perceives, objective support is the level of support available, and support utilization is a measure of support used by the person. Previous studies showed that patients with lower levels of social support had poorer general health than those with higher levels of social support, ${ }^{9}$ and, among persons with diabetes, higher levels of social support were associated with better diabetes self-care behaviors, such as following a diabetes diet plan, caring for feet, exercising, monitoring blood glucose, ${ }^{10}$ and adhering to treatment. ${ }^{11}$ In addition, a high level of social support could mitigate the negative impact of cognitive impairment on glycemic control. ${ }^{12}$

Type D personality has been recognized as a risk factor for a wide range of diseases. ${ }^{13}$ Type $\mathrm{D}$ personality is the interaction of two stable personality traits: negative affectivity (NA), which is the tendency to experience negative emotions across time and situations, and social inhibition (SI), which is the tendency to inhibit the expression of emotions. Patients who score high on NA frequently report feelings of dysphoria, worry, and tension, and patients who score high on SI tend to avoid negative reactions from others. ${ }^{14,15}$ Type D personality captures these two key personality traits and could be considered a potent and easier alternative scale to understand care for patients with diabetes and other diseases for which psychological factors play important roles.

Previous studies showed that Type D personality was predictive of poor health outcomes in patients with cardiovascular disease, ${ }^{13,15-17}$ along with an increased risk of vital exhaustion ${ }^{18}$ and increased odds of impaired health status. ${ }^{19}$ However, research on the association between Type D personality and T2DM has been limited. Our review found only four studies of relevance. Nefs et $\mathrm{al}^{20,21}$ found that T2DM patients with Type D personality experienced less social support and more stressful life events, loneliness, depressed mood, anhedonia, and anxiety. Milicevic et $\mathrm{al}^{22}$ found that T2DM patients with Type D personality were less compliant with visits to primary care physicians. Li et $\mathrm{al}^{23}$ discovered that Type D personality predicted poor medication adherence in T2DM patients. More studies are needed to understand the impact of Type D personality in relation to other important sociopsychological factors in T2DM patients, such as self-efficacy and social support.

In this study, we tried to estimate the prevalence of Type D personality in a sample of Chinese patients with T2DM and assess the relationship between Type D personality and self-efficacy/social support. By establishing a link between Type D personality and the two well-established and wellresearched sociopsychological concepts (ie, self-efficacy and social support), we intended to understand this personality type in the context of diabetic patients and study how to use this information to implement adequate diabetic care.

\section{Patients and methods Settings and participants}

The study was approved by the ethics committee of Guangdong General Hospital. Consecutive participants were recruited among the outpatients and inpatients who visited the endocrine clinics of two of the largest hospitals in Guangzhou City, Guangdong, between January 1, 2014, and July 31, 2014. The participants were provided with both written and oral information regarding the study and were informed that they were free to withdraw from the study at any time.

The following inclusion criteria were applied to the study participants: the patients 1) who were diagnosed as having T2DM according to the American Diabetes Association (ADA) criteria (2010); 2) who had been receiving diabetic treatment for $>1$ year; 3) who had no concurrent malignant tumor, type 1 diabetes, gestational diabetes, vision impairment due to complications, limited physical activity due to advanced renal failure, or acute complications; 4) who were local Chinese inhabitants; 5) who were at least 18 years of age; and 6) who were able and willing to complete the questionnaire alone.

\section{Data and measures}

Once recruited and having provided written consent to participate, the subjects were first asked to complete the general questionnaire containing sociodemographic data in a quiet environment, and then three-scale questionnaires (ie, personality, social support, and self-efficacy scales) under the guidance of trained professionals according to the standard instructions of each scale. There was no mandatorily stipulated time for completing the scale. The patient's medical records were reviewed to retrieve additional information on medical conditions and treatment history. Glycosylated hemoglobin (HbA1c), an integrated marker of glycemic control, was obtained from their medical records if it was measured in the past 3 months; otherwise, blood samples were taken 
after the patients completed the questionnaires, and $\mathrm{HbA} 1 \mathrm{c}$ was tested in the laboratories of the participating hospitals.

We used Type D Scale (DS14) ${ }^{14}$ to assess Type D personality. There are 14 items in the DS14, covering two domains, NA and SI. The former includes item 2 (I often make a fuss about unimportant things), item 4 (I often feel unhappy), item 5 (I often feel irritated), item 7 (I take a gloomy view of things), item 9 (I am often in a bad mood), item 12 (I often find myself worrying about something), and item 13 (I am often down in the dumps). The latter includes item 1 (I make contact easily when I meet people), item 3 (I often talk to strangers), item 6 (I often feel inhibited in social interactions), item 8 (I find it hard to start a conversation), item 10 (I am a closed kind of person), item 11 (I would rather keep other people at a distance), and item 14 (When socializing, I do not find the right things to talk about). The responses are measured on a 5-point Likert scale ranging from 0 (false) to 4 (true). Each of the two subscales includes seven items, with a total score ranging from 0 to 28 . A patient was determined to have Type D personality if both subscales score 10 points or higher.

Both subscales have been shown previously to be internally consistent (Cronbach's $\alpha=0.88$ and 0.86 for NA and SI, respectively). ${ }^{14}$ The Chinese version of the DS14 scale has been tested previously and found to have a similar level of internal consistency (Cronbach's $\alpha=0.90$ and 0.85 for NA and SI, respectively). ${ }^{24}$

We used the self-efficacy scale designed by Lorig et $\mathrm{al}^{25}$ at Stanford University during their research of self-management behavior in patients with chronic diseases. It contains six items reflecting the multiple aspects of self-efficacy in patients with chronic diseases, including emotional control, communication with doctors, symptom management, and role function. Each item is scored from 0 (no confidence at all) to 9 (full confidence). The average score of the six items indicates the level of self-efficacy, with a higher score reflecting better self-efficacy. The Cronbach's $\alpha$ value of this scale was previously estimated at $0.89 .{ }^{25}$

We used the 10-item "Social Support Rating Scale" to assess a patient's social support. ${ }^{26}$ This scale includes three subscales: subjective support (four items), objective support (three items), and the utilization of support (three items), with items scored on either yes-no or 4-point Likert scale. Objective support score is the sum of scores from items 2, 6 , and 7 , subjective support score is the sum of scores from items $1,3,4$, and 5, support utilization score is the sum of scores from items 8,9 , and 10 , and the total social support score is the sum of scores across all 10 items. A higher summary score indicates stronger social support. A study showed that the Cronbach's $\alpha$ of this instrument is between 0.825 and $0.896 . .^{27}$

\section{Statistical analysis}

Data were entered into a database using EpiData 3.1 software. Simultaneous data entry was carried out by two persons (hospital staff or graduate students) with data entry experience, and the consistency check function of the EpiData software was used to ensure accuracy. Once the data were collected and entered into the computer, the Cronbach's $\alpha$ values were calculated to assess the internal consistency of the three scales used. Differences in the sociodemographic characteristics between patients with Type D and non-Type D personalities were compared using the unpaired, two-sided $t$-test (for continuous variables) and the chi-square test (for categorical variables). Bivariate analyses, including the $t$-test and correlation analysis, were used to assess the relationship between Type D personality and self-efficacy/social support scores. Finally, regression analyses were conducted to examine the relationship between Type D personality and self-efficacy/social support while controlling for confounding sociodemographic characteristics. A $P$-value of $<0.05$ was considered statistically significant. All statistical analyses were performed using SPSS 15.0 (IBM, Armonk, NY, USA) for Windows.

\section{Results}

Three-quarters of the participants were recruited from the outpatient departments of the two hospitals. Most of the participants were elderly, female patients. Of the 532 patients in the sample, $97(18.2 \%)$ had Type D personality.

Table 1 lists the sociodemographic characteristics of the patients with and without Type D personality. Overall, there were no significant differences in the distributions of age, gender, education, body mass index (BMI), family size, and income between the two groups, even though the groups with the highest education, largest family size, and highest income appeared to have the lowest percentages of Type D personality. Notably, patients with Type D personality had significantly higher levels of HbAlc than those without Type D personality.

The Cronbach's $\alpha$ values were 0.88 and 0.77 for the two Type D personality subscales, NA and SI, respectively, and 0.89 and 0.60 for self-efficacy and social support scales, respectively. These assessments based on the collected data demonstrate good internal consistency for all three scales in this study.

Table 2 compares the self-efficacy and social support scales in T2DM patients with and without Type D personality. As shown in the table, patients with Type D personality had 
Table I Demographic, social, and clinical characteristics of the participants by Type $D$ personality

\begin{tabular}{|c|c|c|c|}
\hline \multirow[t]{2}{*}{ Variables } & \multirow{2}{*}{$\begin{array}{l}\text { Type D } \\
\mathrm{n}=97\end{array}$} & \multirow{2}{*}{$\frac{\text { Non-Type D }}{n=435}$} & \multirow[t]{2}{*}{$P$-value } \\
\hline & & & \\
\hline Gender (male, n, \%) & $37,38.1$ & $187,43.0$ & 0.224 \\
\hline Age & $61.77 \pm 11.23$ & $63.79 \pm 11.05$ & 0.106 \\
\hline Height (cm) & $160.49 \pm 8.26$ & $|60.4| \pm 8.39$ & 0.896 \\
\hline Weight (kg) & $62.38 \pm 10.72$ & $62.13 \pm 10.48$ & 0.834 \\
\hline BMI & $24.20 \pm 3.70$ & $24.10 \pm 3.7$ & 0.767 \\
\hline Waist circumference $(\mathrm{cm})$ & $87.56 \pm 10.78$ & $88.16 \pm 9.12$ & 0.570 \\
\hline Hip circumference $(\mathrm{cm})$ & $97.35 \pm 8.41$ & $96.80 \pm 7.29$ & 0.520 \\
\hline Waist-hip ratio & $0.90 \pm 0.07$ & $0.91 \pm 0.07$ & 0.096 \\
\hline Inpatient (n, \%) & $19,19.6 \%$ & $115,26.4 \%$ & 0.099 \\
\hline $\begin{array}{l}\text { Systolic blood pressure } \\
(\mathrm{mmHg})\end{array}$ & $|34.26 \pm| 7.5 \mid$ & $|3| .|4 \pm| 6.29$ & 0.093 \\
\hline $\begin{array}{l}\text { Diastolic blood pressure } \\
(\mathrm{mmHg})\end{array}$ & $77.73 \pm 8.92$ & $76.19 \pm 8.72$ & 0.116 \\
\hline $\mathrm{HbAlc} \%$ & $8.25 \pm 1.99$ & $7.85 \pm 1.74$ & 0.043 \\
\hline Educational level (n, \%) & & & 0.355 \\
\hline Primary school or lower & $27,27.8$ & $117,26.9$ & \\
\hline Middle school & $19,19.6$ & $91,20.9$ & \\
\hline High school & $37,38.1$ & $118,27.1$ & \\
\hline $\begin{array}{l}\text { University/college or } \\
\text { higher }\end{array}$ & $\mid 4,14.4$ & $109,25.1$ & \\
\hline \multicolumn{2}{|c|}{ Individual income (monthly, $¥, \mathrm{n}, \%$ ) } & & 0.001 \\
\hline$<1,000$ & $33,34.0$ & $104,23.9$ & \\
\hline $1,000-1,999$ & $40,41.2$ & $160,36.8$ & \\
\hline $2,000-2,999$ & $16,16.5$ & $70,16.1$ & \\
\hline $3,000-3,999$ & $5,5.2$ & $49,11.3$ & \\
\hline$\geq 4,000$ & $3,3.1$ & $52,12.0$ & \\
\hline Family size (n, \%) & & & 0.434 \\
\hline I person & $5,5.2$ & $23,5.3$ & \\
\hline $2-3$ persons & $55,56.7$ & $23 I, 53.1$ & \\
\hline $4-5$ persons & $31,32.0$ & $136,3 \mid .3$ & \\
\hline$\geq 6$ persons & $6,6.2$ & $45,10.3$ & \\
\hline
\end{tabular}

Abbreviation: BMI, body mass index.

significantly lower scores in self-efficacy, total social support, subjective support, and support utilization than patients without Type D personality, but objective support score did not significantly differ between the two groups.

Table 3 further reveals the correlations between the various sociodemographic characteristics, NA, SI, self-efficacy,

Table 2 Self-efficacy and social support scores by Type D personality

\begin{tabular}{lllll}
\hline Variables & \multicolumn{1}{c}{ Type D } & & Non-Type D & P-value \\
\cline { 2 - 2 } & $\mathbf{n}=\mathbf{9 7}$ & & $\mathbf{n}=\mathbf{4 3 5}$ & \\
\hline Self-efficacy & $30.7 \pm 11.3$ & & $39.9 \pm 10.9$ & $<0.001$ \\
Total social support & $33.7 \pm 7.0$ & & $37.8 \pm 7.7$ & $<0.001$ \\
Objective support & $8.4 \pm 3.1$ & & $8.7 \pm 3.3$ & 0.390 \\
Subjective support & $19.2 \pm 4.4$ & & $22.2 \pm 4.9$ & $<0.001$ \\
Degree of support utilization & $6.1 \pm 2.2$ & & $6.9 \pm 2.4$ & 0.003 \\
\hline
\end{tabular}

Note: Data presented as mean \pm standard deviation

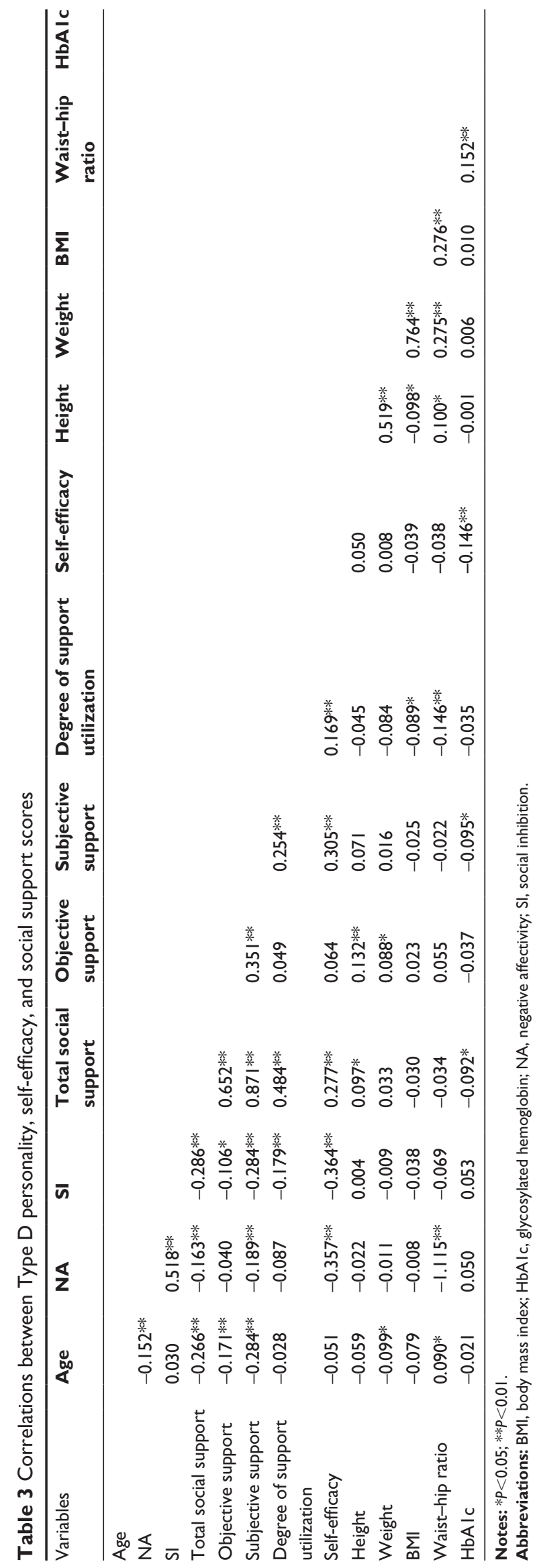


Table 4 Association between Type D personality and selfefficacy/social support

\begin{tabular}{|c|c|c|c|c|}
\hline \multirow[t]{2}{*}{ Variables } & \multicolumn{2}{|l|}{ Self-efficacy } & \multicolumn{2}{|c|}{ Total social support } \\
\hline & Coefficient & $P$-value & Coefficient & $P$-value \\
\hline Type D personality & -9.138 & $<0.001$ & -4.318 & $<0.001$ \\
\hline $\begin{array}{l}\text { Gender, male } \\
\text { (female, ref) }\end{array}$ & 1.093 & 0.274 & 0.307 & 0.640 \\
\hline Age & -0.084 & 0.062 & -0.171 & $<0.001$ \\
\hline BMI & 0.016 & 0.902 & -0.058 & 0.500 \\
\hline \multicolumn{5}{|l|}{ Education } \\
\hline $\begin{array}{l}\text { Primary school } \\
\text { or lower (ref) }\end{array}$ & 1.000 & & 1.000 & \\
\hline Middle school & 1.889 & 0.188 & 0.040 & 0.960 \\
\hline High school & 0.637 & 0.642 & 1.308 & 0.150 \\
\hline $\begin{array}{l}\text { University/college } \\
\text { or higher }\end{array}$ & 1.310 & 0.405 & 0.092 & 0.930 \\
\hline \multicolumn{5}{|l|}{ Family size } \\
\hline I person (ref) & 1.000 & & 1.000 & \\
\hline $2-3$ persons & -0.461 & 0.835 & 3.916 & 0.007 \\
\hline $4-5$ persons & -0.111 & 0.961 & 5.176 & 0.001 \\
\hline$\geq 6$ persons & -2.074 & 0.429 & 4.204 & 0.015 \\
\hline \multicolumn{5}{|c|}{ Personal income (monthly, $¥$ ) } \\
\hline$<\mathrm{I}, 000$ (ref) & 1.000 & & 1.000 & \\
\hline $1,000-1,999$ & -1.214 & 0.339 & 0.958 & 0.250 \\
\hline $2,000-2,999$ & 1.236 & 1.668 & 3.903 & $<0.001$ \\
\hline $3,000-3,999$ & 3.014 & 0.119 & 3.002 & 0.018 \\
\hline$\geq 4,000$ & 1.720 & 0.386 & 3.239 & 0.013 \\
\hline $\begin{array}{l}\text { Outpatient } \\
\text { (inpatient, ref) }\end{array}$ & -0.715 & 0.520 & 0.763 & 0.290 \\
\hline
\end{tabular}

Notes: $¥$, Chinese Yuan, which values at 0.15 US dollar; ref, reference group. Abbreviation: BMI, body mass index.

and social support. As shown in the table, total social support and subjective support significantly correlated with age and NA, but negatively correlated with SI. Objective support also positively correlated with age and negatively correlated with SI. Support utilization showed a strong negative correlation with SI.

Table 4 presents the findings of the regression analysis. Controlling for other characteristics, patients with Type D personality reported significantly lower self-efficacy and social support. In addition, patients who were older, had smaller family size, and had lower income had significantly lower social support, but such characteristics were not significantly associated with self-efficacy.

\section{Discussion}

In this study, we found that $18.2 \%$ of the T2DM patients had Type D personality. This rate is lower than previously reported for healthy Chinese adults (31.9\%) and Chinese patients with coronary heart disease $(31.4 \%) .{ }^{24}$ It is likely that our convenience sample underestimates the prevalence of Type D personality among Chinese patients with T2DM. It is also likely that, because the participants in our study were more advanced in age and had higher educational levels than the average Chinese individuals, they may be more capable of dealing with their emotional problems, causing them to under-report NA and SI.

The current study shows that the presence of Type D personality did not vary significantly by sociodemographic characteristics, which is consistent with the results from previous studies. ${ }^{14,18,20}$ This finding supports the general theory that Type D personality is an inherent personal trait and does not vary according to sociodemographic factors. ${ }^{28}$

As given in Table 1, HbA1c levels were significantly higher in the patients with Type $\mathrm{D}$ personality than those with non-Type $\mathrm{D}$ personality. Blood glucose is an important factor influencing diabetes progression, as evidenced by several previous studies. One prospective study showed that poor blood glucose control led to diabetic nephropathy and diabetic retinopathy. ${ }^{29}$ A study conducted at 215 collaborating centers in 20 countries from Asia, Australasia, Europe, and North America showed a one-fifth reduction in renal complications resulted from intensive control of glucose. ${ }^{30}$ Another study found that, in T2DM patients with blood glucose controlled to the normal level, complications of the eyes and renal injury decreased by $76 \%$ and $35 \%-56 \%$, respectively, and the incidence of microalbuminuria decreased by $33 \%{ }^{31}$ Stratton et $\mathrm{al}^{32}$ estimated that, for every $1 \%$ reduction in $\mathrm{HbA} 1 \mathrm{c}$, the risk of complications decreases by $21 \%$, the incidence of myocardial infarction decreases by $14 \%$, the incidence of microvascular complications decreases by $37 \%$, the incidence of major vascular complications decreases by $40 \%$, and the diabetes-associated mortality rate decreases by $21 \%$. Most recently, van Dooren et $\mathrm{al}^{33}$ showed that Type D personality was associated with higher HbA1c levels. In short, T2DM patients with Type D personality are more likely to have poor glycemic control and, therefore, are at a higher risk of complications and mortality.

Overall, the results of our bivariate and multivariate analyses confirmed our hypothesis that Type D personality predicts lower levels of self-efficacy and social support among T2DM patients. In other words, patients who were classified as having Type D personality experienced lower levels of self-efficacy and social support.

Thus far, only a few studies have specifically investigated the connection between Type D personality and self-efficacy. Mikkelsen and Einarsen ${ }^{34}$ showed that self-efficacy is significantly associated with NA, worry, irritability, coercion, and 
depressive symptoms, which are all salient traits of Type D personality, ${ }^{14,20}$ and the relationship between this type of personality and depression has been clearly established. ${ }^{17,35}$ Therefore, it is likely that the feelings of NA cause such individuals to believe that they lack the confidence, strength, or patience to take control of their diabetic condition and implement appropriate care, and, as a result, they would score lower in self-efficacy. More recent studies revealed that self-efficacy mediated the relationship between Type D personality and physical activity, ${ }^{36}$ and the relationship between Type D and medication adherence. ${ }^{37}$ Our findings are consistent with these previous studies and highlight the negative impact of Type D personality on patient self-efficacy in controlling their medical conditions.

Our findings regarding the lower social support experienced by Type D patients are also in line with the literature. ${ }^{38-40}$ One of the defining features of Type D personality is inhibitions in social interaction and acquisition of social support. This study revealed that patients with Type D personality reported significantly lower levels of subjective support and less support utilization, but similar level of objective support, compared to those of other patients. This finding suggests that, because of SI, patients with Type D personality perceive lower social support (ie, subjective support) and are, therefore, less likely to use such support (ie, support utilization), even though the social support from their families, friends, neighbors, colleagues, and communities available to them is the same as that for other patients.

This study has some limitations. First, the study sample was not representative of Chinese patients with T2DM and the sample size was small. Therefore, our results should not be generalized to the normal population of patients with T2DM or to patients with other chronic conditions in China. In addition, our assessments were based on patient self-reports; therefore, our findings may be subject to various reporting biases.

\section{Conclusion}

The results of the current study indicate that Type D personality is predictive of reduced self-efficacy and social support among patients with T2DM in China. Because selfefficacy and social support play important roles in treatment compliance and self-care, our study highlights the special needs in diabetes patients with Type D personality and extra efforts needed to ensure sufficient patient follow-up and management to achieve long-term glycemic control and prognosis on par with patients with non-Type $\mathrm{D}$ personality.

\section{Acknowledgments}

We would like to thank Dr Huazhang Yang, Head of Endocrinology Department, and all physicians in the diabetes clinics in the two participating hospitals for their collaboration in the data collection. We also thank Dr Chunliu Zhan, at the Agency for Healthcare Research and Quality, for helpful discussions and writing assistance.

\section{Author contributions}

$\mathrm{CW}$ and $\mathrm{YS}$ designed the study and oversaw the survey. YS and HY collected and analyzed the data. YS, HY and CW drafted and revised the manuscript.

\section{Disclosure}

The authors conducted this study as an intramural research at the hospitals at which they are employed; no outside sources of funding were used. The authors report no conflicts of interest in this work.

\section{References}

1. Nouwen A, Winkley K, Twisk J, et al; European Depression in Diabetes (EDID) Research Consortium. Type 2 diabetes mellitus as a risk factor for the onset of depression: a systematic review and meta-analysis. Diabetologia. 2010;53(12):2480-2486.

2. Roy T, Lloyd CE. Epidemiology of depression and diabetes: a systematic review. J Affect Disord. 2012;142(suppl):S8-S21.

3. Nefs G, Pouwer F, Denollet J, Pop V. The course of depressive symptoms in primary care patients with type 2 diabetes: results from the Diabetes, Depression, Type D Personality Zuidoost-Brabant (DiaDDZoB) Study. Diabetologia. 2012;55(3):608-616.

4. Eren I, Erdi O, Sahin M. The effect of depression on quality of life of patients with type II diabetes mellitus. Depress Anxiety. 2008;25(2): 98-106.

5. DeVellis B, DeVellis R. Self-efficacy and health. In: Baum A, Revenson T, Singh J, editors. Handbook of Health Psychology. New York, NY: Psychology Press; 2001:235-247.

6. Cherrington A, Wallston KA, Russell L, Rothman RL. Exploring the relationship between diabetes self-efficacy, depressive symptoms, and glycemic control among men and women with type 2 diabetes. J Behav Med. 2010;33(1):81-89.

7. Al-Khawaldeh OA, Al-Hassan MA, Froelicher ES. Self-efficacy, self-management, and glycemic control in adults with type 2 diabetes mellitus. J Diabetes Complications. 2012;26(1):10-16.

8. Sarkar U, Fisher L, Schillinger D. Is self-efficacy associated with diabetes self-management across race/ethnicity and health literacy? Diabetes Care. 2006;29(4):823-829.

9. Westaway MS, Seager JR, Rheeder P, Van Zyl DG. The effects of social support on health, well-being and management of diabetes mellitus: a black South African perspective. Ethn Health. 2005;10(1):73-89.

10. Brody GH, Kogan SM, Murry VM, Chen YF, Brown AC. Psychological functioning, support for self-management, and glycemic control among rural African American adults with diabetes mellitus Type 2. Health Psychol. 2008;27(1 suppl):S83-S90.

11. Gomes-Villas Boas LC, Foss MC, Freitas MC, Pace AE. Relationship among social support, treatment adherence and metabolic control of diabetes mellitus patients. Rev Lat Am Enfermagem. 2012;20(1):52-58.

12. Okura T, Heisler M, Langa KM. Association between cognitive function and social support with glycemic control in adults with diabetes mellitus. J Am Geriatr Soc. 2009;57(10):1816-1824. 
13. Denollet J, Sys SU, Stroobant N, Rombouts H, Gillebert TC, Brutsaert DL. Personality as independent predictor of long-term mortality in patients with coronary heart disease. Lancet. 1996;347(8999):417-421.

14. Denollet J. DS14: standard assessment of negative affectivity, social inhibition, and Type D personality. Psychosom Med. 2005;67:89-97.

15. Schiffer AA, Smith OR, Pedersen SS, Widdershoven JW, Denollet J. Type D personality and cardiac mortality in patients with chronic heart failure. Int J Cardiol. 2010;142(3):230-235.

16. Denollet J, Pedersen SS, Vrints CJ, Conraads VM. Predictive value of social inhibition and negative affectivity for cardiovascular events and mortality in patients with coronary artery disease: the Type D personality construct. Psychosom Med. 2013;75(9):873-881.

17. Vukovic O, Tosevski DL, Jasovic-Gasic M, et al. Type D personality in patients with coronary artery disease. Psychiatr Danub. 2014;26(1): 46-51.

18. Pedersen SS, Middel B. Increased vital exhaustion among type-D patients with ischemic heart disease. J Psychosom Res. 2001;51(2):443-449.

19. Mols F, Martens EJ, Denollet J. Type D personality and depressive symptoms are independent predictors of impaired health status following acute myocardial infarction. Heart. 2010;96(1):30-35.

20. Nefs G, Pouwer F, Pop V, Denollet J. Type D (distressed) personality in primary care patients with type 2 diabetes: validation and clinical correlates of the DS14 assessment. J Psychosom Res. 2012;72(4): 251-257

21. Nefs G, Speight J, Pouwer F, Pop V, Bot M, Denollet J. Type D personality, suboptimal health behaviors and emotional distress in adults with diabetes: results from Diabetes MILES-The Netherlands. Diabetes Res Clin Prac. 2015;108(1):94-105.

22. Milicevic R, Jaksic N, Aukst-Margetic B, Jakovljevic M. Personality traits and treatment compliance in patients with type 2 diabetes mellitus. Psychiatr Danub. 2015;27(suppl 2):586-589.

23. Li X, Zhang S, Xu H, et al. Type D personality predicts poor medication adherence in Chinese patients with type 2 diabetes mellitus: a six-month follow-up study. PLoS One. 2016;11(2):e0146892.

24. Yua XN, Zhang JX, Liu XH. Application of the Type D Scale (DS14) in Chinese coronary heart disease patients and healthy controls. J Psychosom Res. 2008;65(6):595-601.

25. Lorig K, Stewart A, Ritter P, et al. Outcome Measures for Health Education and other Health Care Interventions. Thousand Oaks CA: Sage Publications; 1996.

26. Shuiyuan X. Social Support Rating Scale (SSRS). Chin Mental Health J. 1999;(suppl):101-103.

27. Liu JW, Li FY, Lian YL. Investigation of reliability and validity of the social support scale. J Xinjiang Med Univ. 2008;31(1):1-3.

28. Kupper N, Boomsma DI, de Geus EJ, Denollet J, Willemsen G. Nine-year stability of type D personality: contributions of genes and environment. Psychosom Med. 2011;73(1):75-82.
29. Wang PH, Lau J, Chalmers TC. Meta-analysis of effects of intensive blood-glucose control on late complications of type I diabetes. Lancet. 1993;341(8856):1306-1309.

30. ADVANCE Collaborative Group; Patel A, MacMahon S, et al. Intensive blood glucose control and vascular outcomes in patients with type 2 diabetes. N Engl J Med. 2008;358(24):2560-2572.

31. UK Prospective Diabetes Study (UKPDS) Group. Intensive bloodglucose control with sulphonylureas or insulin compared with conventional treatment and risk of complications in patients with type 2 diabetes (UKPDS 33). Lancet. 1998;352(9131):837-853.

32. Stratton IM, Adler AI, Neil HA, et al. Association of glycaemia with macrovascular and microvascular complications of type 2 diabetes (UKPDS 35): prospective observational study. BMJ. 2000; 321(7258):405-412.

33. van Dooren FE, Verhey FR, Pouwer F, et al. Association of Type D personality with increased vulnerability to depression: is there a role for inflammation or endothelial dysfunction? - The Maastricht Study. $J$ Affect Disord. 2016;189:118-125.

34. Mikkelsen EG, Einarsen S. Relationships between exposure to bullying at work and psychological and psychosomatic health complaints: the role of state negative affectivity and generalized self-efficacy. Scand $J$ Psychol. 2002;43(5):397-405.

35. Pedersen SS, Herrmann-Lingen C, de Jonge P, Scherer M. Type D personality is a predictor of poor emotional quality of life in primary care heart failure patients independent of depressive symptoms and New York Heart Association functional class. J Behav Med. 2010; 33(1):72-80.

36. Wiencierz S, Williams L. Type D personality and physical inactivity: the mediating effects of low self-efficacy. J Health Psychol. In press 2017. Epub 2016 Feb 1.

37. Wu JR, Song EK, Moser DK. Type D personality, self-efficacy, and medication adherence in patients with heart failure-A mediation analysis. Heart Lung. 2015;44(4):276-281.

38. Sararoudi RB, Sanei H, Baghbanian A. The relationship between type D personality and perceived social support in myocardial infarction patients. J Res Med Sci. 2011;16(5):627-633.

39. Williams L, Wingate A. Type D personality, physical symptoms and subjective stress: the mediating effects of coping and social support. Psychol Health. 2012;27(9):1075-1085.

40. Staniute M, Brozaitiene J, Burkauskas J, Kazukauskiene N, Mickuviene N, Bunevicius R. Type D personality, mental distress, social support and health-related quality of life in coronary artery disease patients with heart failure: a longitudinal observational study. Health Qual Life Outcomes. 2015;13:1.
Neuropsychiatric Disease and Treatment

\section{Publish your work in this journal}

Neuropsychiatric Disease and Treatment is an international, peerreviewed journal of clinical therapeutics and pharmacology focusing on concise rapid reporting of clinical or pre-clinical studies on a range of neuropsychiatric and neurological disorders. This journa is indexed on PubMed Central, the 'PsycINFO' database and CAS,

\section{Dovepress}

and is the official journal of The International Neuropsychiatric Association (INA). The manuscript management system is completely online and includes a very quick and fair peer-review system, which is all easy to use. Visit http://www.dovepress.com/testimonials.php to read real quotes from published authors. 